\title{
Darboux Transformation and New Multi-Soliton Solutions of the Whitham-Broer-Kaup System
}

\author{
Tiantian Xu \\ College of Science, University of Shanghai for Science and Technology, Shanghai, China \\ Email: xutiantian0197@163.com
}

Received 4 November 2014; revised 2 December 2014; accepted 19 December 2014

Copyright (C) 2015 by author and Scientific Research Publishing Inc.

This work is licensed under the Creative Commons Attribution International License (CC BY).

http://creativecommons.org/licenses/by/4.0/

(c) () Open Access

\begin{abstract}
Through a variable transformation, the Whitham-Broer-Kaup system is transformed into a parameter Levi system. Based on the Lax pair of the parameter Levi system, the $\mathrm{N}$-fold Darboux transformation with multi-parameters is constructed. Then some new explicit solutions for the Whitham-Broer-Kaup system are obtained via the given Darboux transformation.
\end{abstract}

\section{Keywords}

Whitham-Broer-Kaup Equation, Levi Parameter System, Lax Pair, Darboux Transformation, Soliton Solutions

\section{Introduction}

Studying of the nonlinear models in shallow water wave is very important, such as Korteweg-de Vries (KdV) equation [1] [2], Kadomtsev-Petviashvili (KP) equation [3] [4], Boussinesq equation [5] [6], etc. There are many methods to study these nonlinear models, such as the inverse scattering transformation [7], the Bäcklund transformation (BT) [8], the Hirota bilinear method [9], the Darboux transformation (DT) [10], and so on. Among those various approaches, the DT is a useful method to get explicit solutions.

In this paper, we investigate the Whitham-Broer-Kaup (WBK) system [11]-[13] for the dispersive long water in the shallow water

$$
\left\{\begin{array}{l}
u_{t}+u u_{x}+v_{x}+\beta u_{x x}=0 \\
v_{t}+(u v)_{x}+\alpha u_{x x x}-\beta v_{x x}=0,
\end{array}\right.
$$

where $u=u(x, t)$ is the field of the horizontal velocity, and $v=v(x, t)$ is the height that deviates from equi- 
librium position of the liquid. The constants $\alpha$ and $\beta$ represent different diffusion powers. If $\alpha=0$ and $\beta \neq 0$, the WBK system (1) reduces to the classical long-wave system that describes the shallow water wave with diffusion [14]. If $\alpha=1$ and $\beta=0$, the WBK system (1) becomes the modified Boussinesq-Burgers equation [7].

Many solutions have been obtained for the WBK system (1), such as the analytical solution, the soliton-like solution, the soliton solutions, the periodic solution, the rational solution, and so on [15]-[19].

In this paper, through a proper transformation

$$
\left\{\begin{array}{l}
u=c\left[\ln \left(r_{x}-q_{x}+q^{2}-r^{2}\right)\right]_{x}-2 c r \\
v=-c^{2}\left(r_{x}-q_{x}+q^{2}-r^{2}\right)-\frac{2 \beta c-c^{2}}{2}\left[\ln \left(r_{x}-q_{x}+q^{2}-r^{2}\right)\right]_{x x}+\left(2 \beta c-c^{2}\right) r_{x},
\end{array}\right.
$$

the WBK system (1) is transformed into the parameter Levi system

$$
\left\{\begin{array}{l}
q_{t}-c(q r)_{x}-\frac{c}{2} r_{x x}=0 \\
r_{t}-\frac{c}{2} q_{x x}-3 c r r_{x}+c q q_{x}=0
\end{array}\right.
$$

Based on the obtained Lax pair, we construct the N-fold DT of the parameter Levi system (3) and then get the $\mathrm{N}$-fold DT of the WBK system (1). Resorting to the obtained DT, we get new multi-soliton solutions of the WBK system.

The paper is organized as follows. In Section 2, we construct the N-fold DT of the Levi system and the WBK system. In Section 3, DT will be applied to generate explicit solutions of the WBK system (1).

\section{Darboux Transformation}

In this section, we first construct the N-fold DT of the parameter Levi system, and then get explicit solutions of the WBK system.

We consider the following spectral problem corresponding to the Levi system (3)

$$
\varphi_{x}=U \varphi, \quad \varphi=\left(\varphi_{1}, \varphi_{2}\right)^{\mathrm{T}}, \quad U=\left(\begin{array}{cc}
\lambda+q & 2 \lambda(r-q) \\
1 & -\lambda-q
\end{array}\right)
$$

and its auxiliary problem

$$
\varphi_{t}=V \varphi, \quad V=\left(\begin{array}{cc}
-c \lambda^{2}+c(r-q) \lambda+\frac{c}{2} r_{x}+c r q & -2 c(r-q) \lambda^{2}+c\left(2 r^{2}-2 r q+q_{x}-r_{x}\right) \lambda \\
-c \lambda+c r & c \lambda^{2}-c(r-q) \lambda-\frac{c}{2} r_{x}-c r q
\end{array}\right),
$$

where $\lambda$ is a spectral parameter and $c^{2}=4\left(\alpha+\beta^{2}\right)$. The compatibility condition $\varphi_{x t}=\varphi_{t x}$ yields a zero curvature equation $U_{t}-V_{x}+U V-V U=0$ which leads to the Levi system (3) by a direct computation.

Now we introduce a transformation of (4) and (5)

$$
\bar{\varphi}=T \varphi,
$$

where $T$ is defined by

$$
T_{x}+T U=\bar{U} T, \quad T_{t}+T V=\bar{V} T .
$$

Then the Lax pair (4) and (5) are transformed into

$$
\begin{aligned}
& \bar{\varphi}_{x}=\bar{U} \bar{\varphi}, \\
& \bar{\varphi}_{t}=\bar{V} \bar{\varphi},
\end{aligned}
$$


where $\bar{U}, \bar{V}$ have the same form as $U, V$, except replacing $q, r, q_{x}, r_{x}$ with $\bar{q}, \bar{r}, \bar{q}_{x}$, $\bar{r}_{x}$, respectively.

In order to make the Lax pair (4) and (5) invariant under the transformation (6), it is necessary to find a matrix $T$.

Let the matrix $T$ in (6) be in the form of

$$
T=T(\lambda)=\alpha\left(\begin{array}{ll}
A(\lambda) & B(\lambda) \\
C(\lambda) & D(\lambda)
\end{array}\right)
$$

with

$$
A(\lambda)=\sum_{k=0}^{N-1} A_{k} \lambda^{k}+\lambda^{N}, \quad B(\lambda)=\sum_{k=0}^{N-1} B_{k} \lambda^{k+1}, \quad C(\lambda)=\sum_{k=0}^{N-1} C_{k} \lambda^{k}, \quad D(\lambda)=\sum_{k=0}^{N-1} D_{k} \lambda^{k},
$$

where $\alpha, A_{k}, B_{k}, C_{k}, D_{k}(0 \leq k \leq N-1)$ are functions of $x$ and $t$.

Let $\varphi\left(\lambda_{j}\right)=\left(\varphi_{1}\left(\lambda_{j}\right), \varphi_{2}\left(\lambda_{j}\right)\right)^{\mathrm{T}}, \psi\left(\lambda_{j}\right)=\left(\psi_{1}\left(\lambda_{j}\right), \psi_{2}\left(\lambda_{j}\right)\right)^{\mathrm{T}}$ be two basic solutions of the spectral problem (4) and use them to define a linear algebraic system

$$
\left\{\begin{array}{l}
\sum_{k=0}^{N-1}\left(A_{k}+B_{k} \delta_{j} \lambda_{j}\right) \lambda_{j}^{k}=-\lambda_{j}^{N}, \\
\sum_{k=0}^{N-1}\left(C_{k}+D_{k} \delta_{j}\right) \lambda_{j}^{k}=0
\end{array}\right.
$$

with

$$
\delta_{j}=\frac{\varphi_{2}\left(\lambda_{j}\right)-r_{j} \psi_{2}\left(\lambda_{j}\right)}{\varphi_{1}\left(\lambda_{j}\right)-r_{j} \psi_{1}\left(\lambda_{j}\right)}, \quad 1 \leq j \leq 2 N-1,
$$

where the constants $\lambda_{j}, r_{j}\left(\lambda_{k} \neq \lambda_{j}, r_{k} \neq r_{j}, k \neq j\right)$ are suitably chosen such that the determinant of the coefficients of (11) are nonzero. If we take

$$
B_{N-1}=r-q, \quad C_{N-1}=\frac{1}{2},
$$

then $A_{k}, B_{k}, C_{k}, D_{k}(0 \leq k \leq N-1)$ are uniquely determined by (11).

From (10), we have

$$
\operatorname{det} T\left(\lambda_{j}\right)=\alpha^{2}\left[A\left(\lambda_{j}\right) D\left(\lambda_{j}\right)-B\left(\lambda_{j}\right) C\left(\lambda_{j}\right)\right] .
$$

We note that (11) can be written as a linear algebraic system

$$
A\left(\lambda_{j}\right)=-\delta_{j} B\left(\lambda_{j}\right), \quad C\left(\lambda_{j}\right)=-\delta_{j} D\left(\lambda_{j}\right)
$$

and

$$
\operatorname{det} T\left(\lambda_{j}\right)=0,
$$

which implies that $\lambda_{j}(1 \leq j \leq 2 N-1)$ are $2 N-1$ roots of $\operatorname{det} T(\lambda)=0$, that is

$$
\operatorname{det} T(\lambda)=\gamma \prod_{j=1}^{2 N-1}\left(\lambda-\lambda_{j}\right),
$$

where $\gamma$ is independent of $\lambda$. From the above facts, we can prove the following propositions.

Proposition 1. Let $\alpha$ satisfy the following first-order differential equation

$$
\partial_{x} \ln \alpha=-\frac{r}{2}-\frac{q}{2}+\left(A_{N-1}-D_{N-1}-2 C_{N-2}\right)+\frac{1}{4 D_{N-1}-2 r+2 q}\left[r_{x}-q_{x}+2 A_{N-1}(r-q)-2 B_{N-2}-r^{2}+q^{2}\right] .
$$


Then the matrix $\bar{U}$ determined by Equation (7) is the same form as $U$ :

$$
\bar{U}=\left(\begin{array}{cc}
\lambda+\bar{q} & 2 \lambda(\bar{r}-\bar{q}) \\
1 & -\lambda-\bar{q}
\end{array}\right),
$$

where the transformations from the old potentials $q, r$ to $\bar{q}, \bar{r}$ are given by

$$
\left\{\begin{array}{l}
\bar{q}=\frac{r-q}{2}+\left(A_{N-1}-D_{N-1}-2 C_{N-2}\right)-\frac{1}{4 D_{N-1}-2 r+2 q}\left[r_{x}-q_{x}+2 A_{N-1}(r-q)-2 B_{N-2}-r^{2}+q^{2}\right], \\
\bar{r}=\frac{r-q}{2}+\left(A_{N-1}-D_{N-1}-2 C_{N-2}\right)+\frac{1}{4 D_{N-1}-2 r+2 q}\left[r_{x}-q_{x}+2 A_{N-1}(r-q)-2 B_{N-2}-r^{2}+q^{2}\right] .
\end{array}\right.
$$

Proof: Let $T^{-1}=T^{*} / \operatorname{det} T$ and

$$
\left(T_{x}+T U\right) T^{*}=\left(\begin{array}{ll}
f_{11}(\lambda) & f_{12}(\lambda) \\
f_{21}(\lambda) & f_{22}(\lambda)
\end{array}\right)
$$

where $T^{*}$ denotes the adjoint matrix of $T$. It is easy to see that $f_{11}(\lambda)$ and $f_{22}(\lambda)$ are $2 N$ th-order polynomials in $\lambda$, while $\lambda^{-1} f_{12}(\lambda), f_{21}(\lambda)$ are (2N-1)th-order polynomials in $\lambda$. From (4) and (12), we get

$$
\delta_{j x}=1-2\left(\lambda_{j}+q\right) \delta_{j}-2 \lambda_{j}(r-q) \delta_{j}^{2} .
$$

By using (16) and (20), we can prove that $\lambda_{j}(1 \leq j \leq 2 N-1)$ are the roots of $f_{k j}(\lambda)(k, j=1,2)$. From (15), we have

$$
\operatorname{det} T \mid f_{k j}(\lambda), \quad k, j=1,2
$$

Hence, together with (19), we have

$$
\left(T_{x}+T U\right) T^{*}=(\operatorname{det} T) P(\lambda)
$$

that is

$$
T_{x}+T U=P(\lambda) T
$$

with

$$
P(\lambda)=\left(\begin{array}{cc}
p_{11}^{(1)} \lambda+p_{11}^{(0)} & p_{12}^{(1)} \lambda \\
p_{21}^{(1)} \lambda & p_{22}^{(1)} \lambda+p_{22}^{(0)}
\end{array}\right),
$$

where $p_{k j}^{(l)}(k, j=1,2, l=0,1)$ are independent of $\lambda$. By comparing the coefficients of $\lambda^{N+1}, \lambda^{N}$ and $\lambda^{N-1}$ in (22), we find

$$
\begin{gathered}
p_{11}^{(1)}=-p_{22}^{(1)}=1, \quad p_{21}^{(0)}=1, \\
p_{12}^{(1)}=\frac{2}{2 D_{N-1}-r+q}\left[r_{x}-q_{x}+2 A_{N-1}(r-q)-2 B_{N-2}-r^{2}+q^{2}\right], \\
p_{11}^{(0)}=\partial_{x} \ln \alpha+r-\frac{1}{2} p_{12}^{(1)}, \\
p_{22}^{(0)}=\partial_{x} \ln \alpha+4 C_{N-2}+2 D_{N-1}-2 A_{N-1}+q .
\end{gathered}
$$

Substituting (17) into (24)-(26) yields

$$
p_{12}^{(1)}=2(\bar{r}-\bar{q}), \quad p_{11}^{(0)}=\bar{q}, \quad p_{22}^{(0)}=-\bar{q} .
$$


From (7) and (22), we find that $\bar{U}=P(\lambda)$. The proof is completed. $\square$

Remark. When $N=1$, assuming that $A_{-1}=B_{-1}=C_{-1}=D_{-1}=0$, the DT can be rewritten as

$$
\begin{aligned}
& \bar{q}=\frac{r}{2}-\frac{q}{2}+\left(A_{0}-D_{0}\right)-\frac{1}{4 D_{0}-2 r+2 q}\left[r_{x}-q_{x}+2 A_{0}(r-q)-r^{2}+q^{2}\right], \\
& \bar{r}=\frac{r}{2}-\frac{q}{2}+\left(A_{0}-D_{0}\right)+\frac{1}{4 D_{0}-2 r+2 q}\left[r_{x}-q_{x}+2 A_{0}(r-q)-r^{2}+q^{2}\right] .
\end{aligned}
$$

Let the basic solution $\varphi\left(\lambda_{j}\right), \psi\left(\lambda_{j}\right)$ of (4) satisfy (5) as well. Through a similar way as Proposition 1, we can prove that $\bar{V}$ has the same form as $V$ under the transformation (6) and (18). We get the following proposition.

Proposition 2. Suppose $\alpha$ satisfy the following equation

$$
\partial_{t} \ln \alpha=c\left[(\bar{r}-\bar{q})\left(A_{N-1}-2 C_{N-2}\right)+\frac{1}{2}\left(\bar{q}_{x}-\bar{r}_{x}\right)-A_{N-1}(r-q)+\bar{r}^{2}-r^{2}-\frac{1}{2} r_{x}+B_{N-2}\right] .
$$

Then the matrix $\bar{V}$ defined by (9) has the same form as $V$, that is

$$
\bar{V}=\left(\begin{array}{cc}
-c \lambda^{2}+c(\bar{r}-\bar{q}) \lambda+\frac{c}{2} \bar{r}_{x}+c \overline{r q} & -2 c(\bar{r}-\bar{q}) \lambda^{2}+c\left(2 \bar{r}^{2}-2 \overline{r q}+\bar{q}_{x}-\bar{r}_{x}\right) \lambda \\
-c \lambda+c \bar{r} & c \lambda^{2}-c(\bar{r}-\bar{q}) \lambda-\frac{c}{2} \bar{r}_{x}-c \overline{r q}
\end{array}\right),
$$

where $\bar{q}$ and $\bar{r}$ are given by (18).

The proof of Proposition 2 is similar with Proposition 1, but it is much more tedious and then we omit the proof for brevity. For the similar proof we can also refer to [20] [21].

According to Proposition 1 and 2, the Lax pair (4) and (5) is transformed into the Lax pair (8) and (9), then the transformation (6) and (18): $(\varphi ; q, r) \rightarrow(\bar{\varphi} ; \bar{q}, \bar{r})$ is called the DT of the Lax pair (4) and (5). The Lax pair leads to the parameter Levi system (3) and then the transformation (6) and (18): $(\varphi ; q, r) \rightarrow(\bar{\varphi} ; \bar{q}, \bar{r})$ is also called DT of the parameter Levi system (3). On the other hand, together with the transformation (2), the parameter Levi system (3) is transformed into the WBK system (1), then we get the solutions of the WBK system (1).

Theorem 1. If $(q, r)$ is a solution of the parameter Levi system (3), $(\bar{q}, \bar{r})$ with

$$
\left\{\begin{array}{l}
\bar{q}=\frac{r-q}{2}+\left(A_{N-1}-D_{N-1}-2 C_{N-2}\right)-\frac{1}{4 D_{N-1}-2 r+2 q}\left[r_{x}-q_{x}+2 A_{N-1}(r-q)-2 B_{N-2}-r^{2}+q^{2}\right], \\
\bar{r}=\frac{r-q}{2}+\left(A_{N-1}-D_{N-1}-2 C_{N-2}\right)+\frac{1}{4 D_{N-1}-2 r+2 q}\left[r_{x}-q_{x}+2 A_{N-1}(r-q)-2 B_{N-2}-r^{2}+q^{2}\right] .
\end{array}\right.
$$

is another solution of the parameter Levi system (3), where $A_{N-1}, B_{N-2}, C_{N-2}, D_{N-1}$ are determined by (11) and (13).

From the transformation (2), we find that

Theorem 2. If $(u, v)$ is a solution of the WBK system $(1),(\bar{u}, \bar{v})$ with

$$
\left\{\begin{array}{l}
\bar{u}=c\left[\ln \left(\bar{r}_{x}-\bar{q}_{x}+\bar{q}^{2}-\bar{r}^{2}\right)\right]_{x}-2 c \bar{r}, \\
\bar{v}=-c^{2}\left(\bar{r}_{x}-\bar{q}_{x}+\bar{q}^{2}-\bar{r}^{2}\right)-\frac{2 \beta c-c^{2}}{2}\left[\ln \left(\bar{r}_{x}-\bar{q}_{x}+\bar{q}^{2}-\bar{r}^{2}\right)\right]_{x x}+\left(2 \beta c-c^{2}\right) \bar{r}_{x},
\end{array}\right.
$$

is another solution of the WBK system (1), where $(\bar{q}, \bar{r})$ is determined by (30). Then the transformation $(\varphi ; q, r) \rightarrow(\bar{\varphi} ; \bar{q}, \bar{r})$ is also called the DT of the WBK system (1).

\section{New Solutions}

In this section, we take a trivial solution $(q, r)=(0,1)$ as the "seed" solution, to obtain multi-soliton solutions 
of the WBK system (1).

Substituting $(q, r)=(0,1)$ into the Lax pair (4) and (5), the two basic solutions are

$$
\varphi\left(\lambda_{j}\right)=\left(\begin{array}{c}
\cosh \xi_{j} \\
-\frac{1}{2} \cosh \xi_{j}+\frac{k_{j}}{2 \lambda_{j}} \sinh \xi_{j}
\end{array}\right), \quad \psi\left(\lambda_{j}\right)=\left(\begin{array}{c}
\sinh \xi_{j} \\
-\frac{1}{2} \sinh \xi_{j}+\frac{k_{j}}{2 \lambda_{j}} \cosh \xi_{j}
\end{array}\right)
$$

with $\xi_{j}=k_{j}\left[x-c\left(\lambda_{j}-1\right) t\right], \quad k_{j}=\sqrt{\lambda_{j}^{2}+2 \lambda_{j}} \quad(1 \leq j \leq 2 N-1)$.

According to (12), we get

$$
\delta_{j}=-\frac{1}{2}+\frac{k_{j}}{2 \lambda_{j}}\left(\frac{\tanh \xi_{j}-r_{j}}{1-r_{j} \tanh \xi_{j}}\right), \quad 1 \leq j \leq 2 N-1
$$

For simplicity, we discuss the following two cases, i.e. $N=1$ and $N=2$.

As $N=1$, let $\lambda=\lambda_{1}$, solving the linear algebraic system (11) and (13), we have

$$
A_{0}=-\lambda_{1}-\delta_{1} \lambda_{1}, \quad D_{0}=-\frac{1}{2 \delta_{1}}
$$

according to (28), we get

$$
\bar{q} \triangleq \bar{q}[1]=\frac{1+2\left(1-\lambda_{1}\right) \delta_{1}-6 \lambda_{1} \delta_{1}^{2}-4 \lambda_{1} \delta_{1}^{3}}{2 \delta_{1}\left(1+\delta_{1}\right)}, \quad \bar{r} \triangleq \bar{r}[1]=\frac{1+2\left(1-\lambda_{1}\right) \delta_{1}+2\left(1-\lambda_{1}\right) \delta_{1}^{2}}{2 \delta_{1}\left(1+\delta_{1}\right)} .
$$

Substituting (35) into (31), we obtain the solution of the WBK system (1) as

$$
\left\{\begin{array}{l}
\bar{u}[1]=c[\ln \bar{w}[1]]_{x}-2 c \bar{r}[1] \\
\bar{v}[1]=-c^{2}(\bar{w}[1])-\frac{2 \beta c-c^{2}}{2}[\ln \bar{w}[1]]_{x x}+\left(2 \beta c-c^{2}\right) \bar{r}[1]_{x}
\end{array}\right.
$$

with $\bar{w}[1]=-\frac{2\left(1+\lambda_{1}\right) \delta_{1}+\left(1+2 \lambda_{1}\right) \delta_{1}^{2}}{\left(1+\delta_{1}\right)^{2}}$.

By choosing proper parameters (such as $r_{1}=5, \quad \lambda_{1}=7, c=1, \quad \beta=1 / 15$ ), we find that $\bar{u}[1]$ is a bell-typesoliton and $\bar{v}[1]$ is a M-type-soliton.

As $N=2$, let $\lambda=\lambda_{j}(j=1,2,3)$, together with (11) and (13), we have

$$
A_{1}=\frac{\Delta_{A_{1}}}{\Delta_{1}}, \quad B_{0}=\frac{\Delta_{B_{0}}}{\Delta_{1}}, \quad C_{0}=\frac{\Delta_{C_{0}}}{\Delta_{2}}, \quad D_{1}=\frac{\Delta_{D_{1}}}{\Delta_{2}}
$$

with

$$
\begin{array}{cl}
\Delta_{1}=\left|\begin{array}{lll}
1 & \delta_{1} \lambda_{1} & \lambda_{1} \\
1 & \delta_{2} \lambda_{2} & \lambda_{2} \\
1 & \delta_{3} \lambda_{3} & \lambda_{3}
\end{array}\right|, \quad \Delta_{A_{1}}=\left|\begin{array}{ccc}
1 & \delta_{1} \lambda_{1} & -\lambda_{1}^{2}-\delta_{1} \lambda_{1}^{2} \\
1 & \delta_{2} \lambda_{2} & -\lambda_{2}^{2}-\delta_{2} \lambda_{2}^{2} \\
1 & \delta_{3} \lambda_{3} & -\lambda_{3}^{2}-\delta_{3} \lambda_{3}^{2}
\end{array}\right|, \quad \Delta_{B_{0}}=\left|\begin{array}{lll}
1 & -\lambda_{1}^{2}-\delta_{1} \lambda_{1}^{2} & \lambda_{1} \\
1 & -\lambda_{2}^{2}-\delta_{2} \lambda_{2}^{2} & \lambda_{2} \\
1 & -\lambda_{3}^{2}-\delta_{3} \lambda_{3}^{2} & \lambda_{3}
\end{array}\right|, \\
\Delta_{2}=\left|\begin{array}{lll}
1 & \delta_{1} & \delta_{1} \lambda_{1} \\
1 & \delta_{2} & \delta_{2} \lambda_{2} \\
1 & \delta_{3} & \delta_{3} \lambda_{3}
\end{array}\right|, \quad \Delta_{D_{1}}=\left|\begin{array}{ccc}
1 & \delta_{1} & -\frac{1}{2} \lambda_{1} \\
1 & \delta_{2} & -\frac{1}{2} \lambda_{2} \\
1 & \delta_{3} & -\frac{1}{2} \lambda_{3}
\end{array}\right|, \quad \Delta_{C_{0}}=\left|\begin{array}{ccc}
-\frac{1}{2} \lambda_{1} & \delta_{1} & \delta_{1} \lambda_{1} \\
-\frac{1}{2} \lambda_{2} & \delta_{2} & \delta_{2} \lambda_{2} \\
-\frac{1}{2} \lambda_{3} & \delta_{3} & \delta_{3} \lambda_{3}
\end{array}\right| .
\end{array}
$$

With the help of (30), we get 


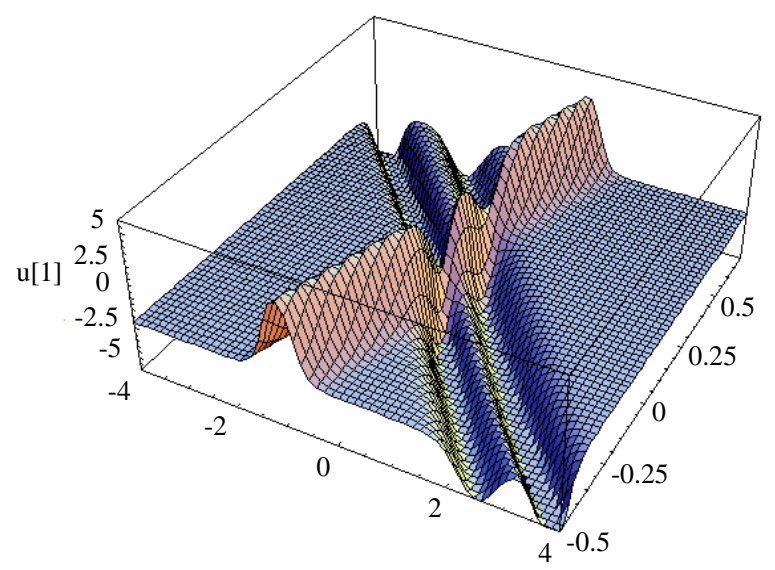

(a)

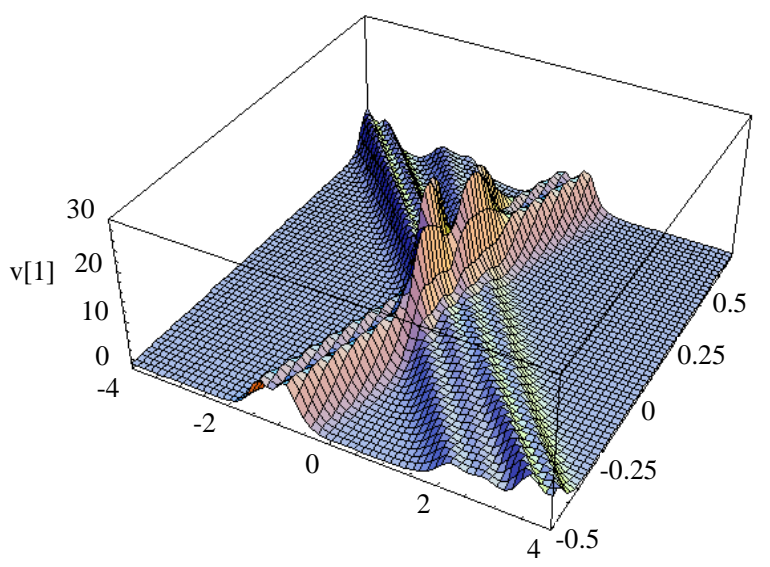

(b)

Figure 1. Plots of the three-soliton solution (39).

$$
\begin{aligned}
& \bar{q} \triangleq \bar{q}[2]=\frac{1}{2}+A_{1}-D_{1}-2 C_{0}-\frac{2 A_{1}-2 B_{0}-1}{4 D_{1}-2}, \\
& \bar{r} \triangleq \bar{r}[2]=\frac{1}{2}+A_{1}-D_{1}-2 C_{0}+\frac{2 A_{1}-2 B_{0}-1}{4 D_{1}-2} .
\end{aligned}
$$

Then we get another solution of the WBK system (1) by using of (31)

$$
\left\{\begin{array}{l}
\bar{u}[2]=c[\ln \bar{w}[2]]_{x}-2 c \bar{r}[2] \\
\bar{v}[2]=-c^{2} \bar{w}[2]-\left(\beta c-\frac{c^{2}}{2}\right)[\ln \bar{w}[2]]_{x x}+\left(2 \beta c-c^{2}\right) \bar{r}[2]_{x}
\end{array}\right.
$$

with $\bar{w}[2]=\bar{r}[2]_{X}-\bar{q}[2]_{X}+\bar{q}[2]^{2}-\bar{r}[2]^{2}$.

When we take $\lambda_{1}=-5, \lambda_{2}=-4, \lambda_{3}=3, c=1, \beta=1 / 20, r_{1}=3, r_{2}=1 / 2, r_{3}=2, \bar{u}[2]$ is a threebell-type-soliton solution with two overtaking solitons and one head-on soliton (see Figure 1(a)) and $\bar{v}$ [2] is a three- $M$-type-soliton solution with two overtaking solitons and one head-on soliton (see Figure 1(b)). We note that by the obtained DT, we can get $(2 N-1)$ soliton solutions which are different from those in [19] which are $2 N$-soliton solutions.

\section{Acknowledgements}

This work is supported by Nurture Funds of National Project of University of Shanghai for Science and Technology (no. 14XPQ09).

\section{References}

[1] Zabusky, N.J. and Galvin, C.J. (1971) Shallow-Water Waves, the Korteweg-de Vries Equation and Solitons. Journal of Fluid Mechanics, 47, 811-824. http://dx.doi.org/10.1017/S0022112071001393

[2] Dullin, H.R., Georg, A.G. and Holm, D.D. (2003) Camassa-Holm, Korteweg-de Vries-5 and Other Asymptotically Equivalent Equations for Shallow Water Waves. Fluid Dynamics Research, 33, 73-95. http://dx.doi.org/10.1016/S0169-5983(03)00046-7

[3] Chakravarty, S. and Kodama, Y. (2009) Soliton Solutions of the KP Equation and Application to Shallow Water Waves. Studies in Applied Mathematics, 123, 83-151. http://dx.doi.org/10.1111/j.1467-9590.2009.00448.x

[4] Kodama, Y. (2010) KP Solitons in Shallow Water. Journal of Physical A: Mathematical and Theoretical, 43, Article ID: 434004. http://dx.doi.org/10.1088/1751-8113/43/43/434004

[5] Lambert, F., Musette, M. and Kesteloot, E. (1987) Soliton Resonances for the Good Boussinesq Equation. Inverse Problems, 3, 275-288. http://dx.doi.org/10.1088/0266-5611/3/2/010

[6] Li, Y.S. and Zhang, J.E. (2001) Darboux Transformation of Classical Boussinesq System and Its Multi-Soliton Solu- 
tions. Physics Letters A, 284, 253-258. http://dx.doi.org/10.1016/S0375-9601(01)00331-0

[7] Ablowitz, M.J. and Clarkson, P.A. (1991) Solitons, Nonlinear Evolution Equations and Inverse Scattering. Cambridge University Press, Cambridge. http://dx.doi.org/10.1017/CBO9780511623998

[8] Rogers, C. and Schief, W.K. (2002) Bäcklund and Darboux Transformations Geometry and Modern Application in Soliton Theory. Cambridge University Press, Cambridge. http://dx.doi.org/10.1017/CBO9780511606359

[9] Hirota, R. (2004) The Direct Method in Soliton Theory. Cambridge University Press, Cambridge. http://dx.doi.org/10.1017/CBO9780511543043

[10] Gu, C.H., Hu, H.S. and Zhou, Z.X. (2005) Darboux Transformation in Soliton Theory and Its Geometric Applications. Shanghai Science Technology Publication House, Shanghai.

[11] Whitham, G.B. (1967) Variational Methods and Applications to Water Wave. Proceedings of the Royal Society A, 299, 6-25. http://dx.doi.org/10.1098/rspa.1967.0119

[12] Broer, L.J. (1975) Approximate Equations for Long Water Waves. Applied Scientific Research, 31, 377-395. http://dx.doi.org/10.1007/BF00418048

[13] Kaup, D.J. (1975) A Higher-Order Water Equation and Method for Solving It. Progress of Theoretical Physics, 54, 396-408. http://dx.doi.org/10.1143/PTP.54.396

[14] Kupershmidt, B.A. (1985) Mathematics of Dispersive Water Waves. Communications in Mathematical Physics, 99, 51-73. http://dx.doi.org/10.1007/BF01466593

[15] Xia, Z. (2004) Homogenous Balance Method and Exact Analytical Solutions for Whitham-Broer-Kaup Equations in the Shallow Water. Chinese Quarterly Journal of Mathematics, 19, 240-246.

[16] Xie, F.D. and Gao, X.S. (2004) A Computational Approach to the New Type Solutions of Whitham-Broer-Kaup Equation in Shallow Water. Communications in Theoretical Physics, 41, 179-182. http://dx.doi.org/10.1088/0253-6102/41/2/179

[17] Zhang, J.F., Guo, G.P. and Wu, F.M. (2002) New Multi-Soliton Solutions and Travelling Wave Solutions of the Dispersive Long-Wave Equations. Chinese Physics, 11, 533-536. http://dx.doi.org/10.1088/1009-1963/11/6/303

[18] Lin, G.D., Gao, Y.T., Gai, X.L. and Meng, D.X. (2011) Extended Double Wronskian Solutions to the Whitham-BroerKaup Equations in Shallow Water. Nonlinear Dynamics, 64, 197-206. http://dx.doi.org/10.1007/s11071-010-9857-5

[19] Wang, L., Gao, Y.T. and Gai, X.T. (2012) Gauge Transformation, Elastic and Inelastic Interactions for the WhithamBroer-Kaup Shallow-Water Model. Communications in Nonlinear Science and Numerical Simulation, 17, 2833-2844. http://dx.doi.org/10.1016/j.cnsns.2011.11.018

[20] Geng, X.G. and Tam, H.W. (1999) Darboux Transformation and Soliton Solutions for Generalized Nonlinear Schrödinger Equations. Journal of Physical Society of Japan, 68, 1508-1512. http://dx.doi.org/10.1143/JPSJ.68.1508

[21] Huang, D.J. and Zhang, H.Q. (2008) Vandermonde-Like Determinants' Representations of Darboux Transformations and Expliclt Solutions for the Modified Kadomtsev-Petviashvili Equation. Physica A, 387, 4565-4580. 
Scientific Research Publishing (SCIRP) is one of the largest Open Access journal publishers. It is currently publishing more than 200 open access, online, peer-reviewed journals covering a wide range of academic disciplines. SCIRP serves the worldwide academic communities and contributes to the progress and application of science with its publication.

Other selected journals from SCIRP are listed as below. Submit your manuscript to us via either submit@scirp.org or Online Submission Portal.
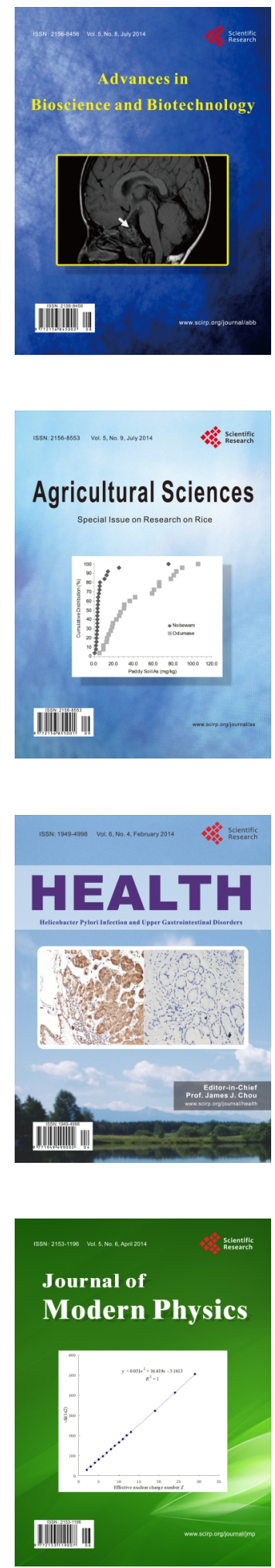
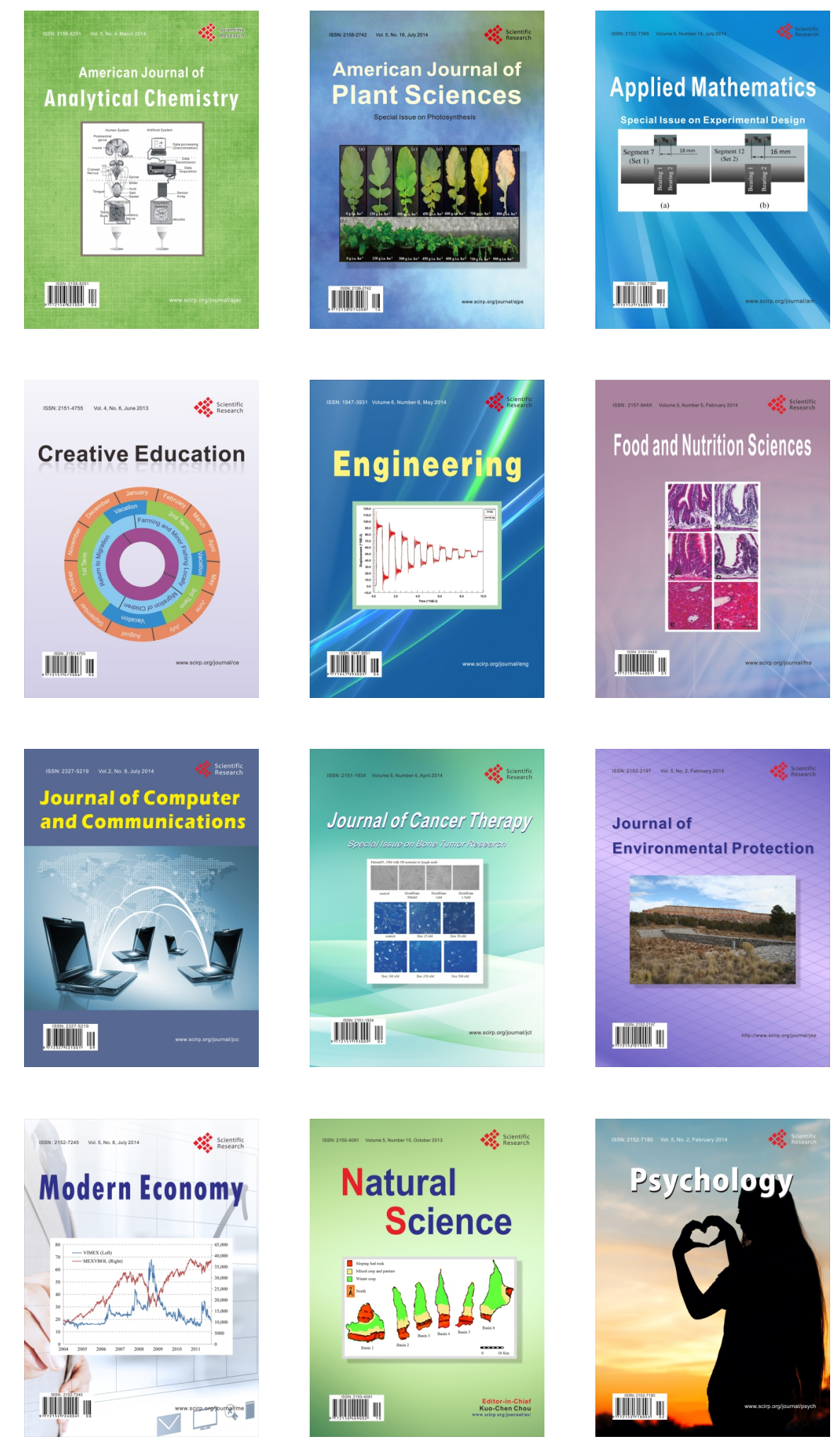